CLAWAR 2020: 23rd International Conference on

Climbing and Walking Robots and the Support

Technologies for Mobile Machines,

Moscow, Russian Federation, 24-26 August 2020.

https://doi.org/10.13180/clawar.2020.24-26.08.38

\title{
Online Adaptive Resistance Control of An Arm Exercise Exoskeleton
}

\author{
XIAOFENG XIONG ${ }^{1}$ and PORAMATE MANOONPONG ${ }^{1,2}$ \\ 1 Embodied AI and Neurorobotics Lab, SDU Biorobotics, the Marsk Mc-Kinney Møller Institute, the \\ University of Southern Denmark (SDU), \\ Odense, 5230, Denmark \\ ${ }^{2}$ Bio-Inspired Robotics and Neural Engineering Lab, the School of Information Science and Technology, \\ Vidyasirimedhi Institute of Science and Technology, \\ Rayong, 21210, Thailand \\ E-mail: xizi@mmmi.sdu.dk,poma@mmmi.sdu.dk \\ https://ens-lab.sdu.dk/
}

\begin{abstract}
Resistance training is an effective means of strengthening musculoskeletal capabilities; thereby preventing musculoskeletal disorders (MSDs). However, most existing exoskeletons fail to provide active and affordable resistance training. To address this problem, we develop a resistance-asneeded (RAN) controller and an arm exercise (RISE) exoskeleton for resistance training. The exoskeleton is designed and manufactured in a portable, lightweight $(0.425 \mathrm{~kg})$, and $3 \mathrm{D}$ print manner. Such control adaptability and design portability are facilitated by the RAN controller whose implementation relies only on internal (e.g., joint position) sensing, rather than external (i.e., EMG, electromyography) sensing and physical compliant mechanisms (e.g., springs). The active resistance torque to the RISE exoskeleton is generated by the RAN controller, in which impedance gains $K_{p}$ and $K_{d}$ are online tuned. As a result, the RAN controller enables the RISE exoskeleton to produce more adaptive and effective resistance motions that lead to higher training efficiency, compared to conventional constant impedance controllers. The proposed RAN controller and designed RISE exoskeleton pave a way for providing portable and affordable resistance training.
\end{abstract}

\section{Introduction}

At-home healthcare is one of keys to solve societal challenges, e.g., high rehabilitation costs and heavy hospitalization burdens [1]. The healthcare problems have been raised mainly by musculoskeletal disorders (MSDs) owing to increasing work-related injuries [2]. Resistance training is regarded as an effective method to reduce the MSDs [3]. Because of their costeffectiveness, robotic devices (e.g., exoskeletons) have shown potentials to provide resistance training $[4,5]$.

However, most existing researches are characterized by bulky actuation, multiple sensing integration, and fixed impedance control $[4,5]$. For example, physical compliant mechanisms (e.g., springs) are added into actuators for emulating muscle-like properties (e.g., compliance) that lead to friendly resistance training. Multiple sensing (e.g., EMG and force) integration requires advanced machine learning algorithms to predict wearers' intentions. However, their signals are inherently noisy and varied over different wearers. Moreover, an activated muscle contributes to various limb movements, therefore leading to inaccurate predictions of wearers' intentions using EMG sensing. Furthermore, the additional sensing becomes imprecise when wearers sweat and sensors fall out. Therefore, such additional sensing decreases exoskeletons' portability, affordability, and adaptivity. Moreover, there is no available resistance-as-needed (RAN) controller for portable arm exercise exoskeletons [5]. Extensive reviews on exoskeleton actuation and control can be seen in [4,5], while recent related works are presented in section 4 . Taken together, most existing exoskeletons fail to live up to at-home resistance training due to their mechatronic and control complexities [4]. Here such training is characterized by the portability, affordability, and wearability of exoskeletons (see section 2). In this paper, we focus on simple actuation, manufacture, sensing, 
and advanced control of a portable arm exoskeleton in resistance training.

Specifically, we propose an online RAN controller to control a portable and lightweight $(0.425 \mathrm{~kg})$ arm exercise (RISE) exoskeleton in resistance training. The RAN controller produces a resistance torque to the exoskeleton by online tuning the impedance gains $K_{p}$ and $K_{d}$ based on joint position and velocity deviations. As a result, the RAN control leads to more stable control and higher training efficiency, compared to conventional impedance controllers. The RAN implementation does not rely on external (e.g., force) sensing and physical compliant components (e.g., springs) owing to advanced software control. This character greatly reduces sensory dependency and actuation complexity. The proposed RAN controller and RISE exoskeleton aim at strengthening human arms such as bicep and tricep muscles. The main contributions of the proposed controller and design are as follows:

- The first novel resistance-as-needed (RAN) exoskeleton controller. It leads to more stable control and higher exercise efficiency, compared to conventional constant impedance control [6-8] (see Figs. 4 and 5).

- A designed exercise exoskeleton is compact, portable, and lightweight $(0.425 \mathrm{~kg})$ (see Fig. 1).

- A quantitative measurement for selecting a proper exoskeleton actuator based on its portability and capability (see Eq. (1) and Fig. 2).

To the best of our knowledge, the proposed RAN and design are the first online adaptive resistance controller and portable exercise exoskeleton, which pave a way for achieving active and portable resistance training. The remainder of this paper is structured as follows. The RISE exoskeleton design and RAN controller are presented in section 2. Section 3 contains the experimental results of adaptive resistant exoskekelton motions generated by the RAN controller, compared to conventional impedance controllers. A discussion and conclusion, including a review of related works, are provided in section 4 .

\section{Design and Control of An Arm Exercise Exoskeleton}

\subsection{Mechatronic Design}

The portable and lightweight $(0.425 \mathrm{~kg})$ arm exercise (RISE) exoskeleton consists of 3D printed carbon fiber reinforced polymer (CFRP) parts, aluminium connectors, and a Dynamixel actuator (see Fig. 1 (A) and Table 1). In addition to the mechanical structure, the portable RISE exoskeleton consists of an interface (i.e., U2D2 ${ }^{\mathrm{a}}$ ) between $\mathrm{PC}$ and the actuator, as well as a power converter (i.e., SMPS ${ }^{\mathrm{b}}$ ) (see Fig. 1 (B)). The U2D2 interface allows the RAN controller ran on PC to send actuator commands and receive joint feedback (i.e., position and velocity).

Table 1. RISE exoskeleton specification

\begin{tabular}{lcccc}
\hline $\begin{array}{l}\text { Weight } \\
{[\mathrm{kg}]}\end{array}$ & Actuator & $\begin{array}{c}\text { Stall torque } \\
{[N \cdot m]}\end{array}$ & $\begin{array}{c}\text { Input voltage and current } \\
{[V] \text { and }[A]}\end{array}$ & Attachment \\
\hline 0.425 & $\mathrm{XM} 430^{\mathrm{a}}$ & 4.1 & 12.0 and 2.3 & Hook-and-loop straps \\
\hline Note: ${ }^{\text {a }}$ http://emanual.robotis.com/docs $/ \mathrm{en} / \mathrm{dxl} / \mathrm{x} / \mathrm{xm} 430-\mathrm{w} 350 /$ &
\end{tabular}

The simple and compact hardware design is easy to manufacture and wear. An exoskeleton portability depends mainly on its actuators [4]. To reduce mechatronic complexity, a

\footnotetext{
${ }^{a}$ http://emanual.robotis.com/docs/en/parts/interface/u2d2/

${ }^{b}$ http://www.robotis.us/smps2dynamixel/
} 

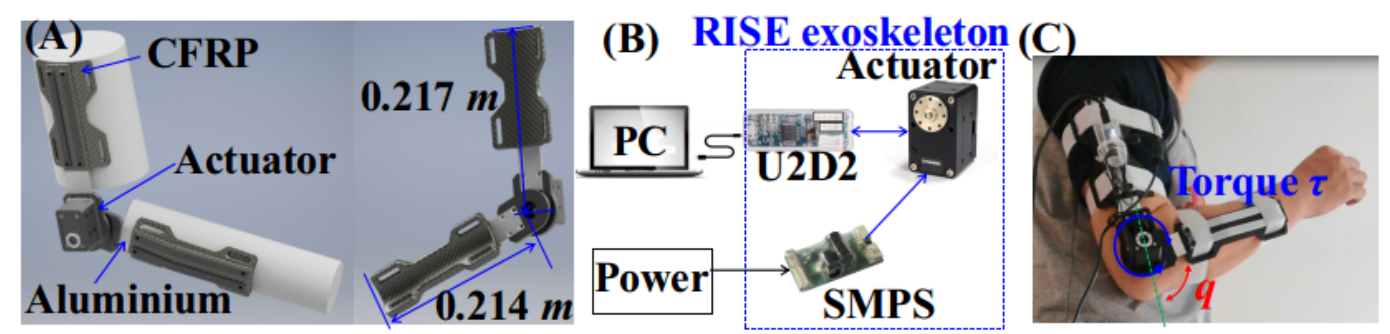

Fig. 1. Portable arm exercise (RISE) exoskeleton mechatronic system. (A) Mechanical structure. CFRP: carbon fiber reinforced polymer. (B) Electronic design. (C) Exoskeleton wearer. $q$ is the joint angle.

simple and all-in-one actuator should be chosen. 'All-in-one' means that all actuator components such as a motor and sensors are integrated into the actuator except a power supply and communication interface. In addition, the weight and price are other key factors to choose actuators in terms of at-home exoskeleton portability and affordability. Most existing actuators are very heavy (i.e., $>0.2 \mathrm{~kg}$ ) and usually used in ground-based exoskeletons. By contrast, body-based (i.e., portable) and at-home exoskeletons require lightweight and affordable actuators. Exoskeleton safety can be guaranteed by choosing proper actuator stall torques (i.e., $<8 N \cdot m$ ) and torque-based (i.e., impedance) control, rather than positionbased (e.g., admittance) control. Taken together, a criterion of choosing a portable and cost-effective exoskeleton actuator is summarized in TABLE 2. According to the criterion, a Dynamixel actuator is chosen for the RISE exoskeleton. This is because it is a lightweight and affordable actuator integrating a DC (Direct Current) motor, reduction gearhead, controller, driver, and network. Choosing a proper Dynamixel actuator for a portable exoskeleton is quantified by its weight to torque $(W T)$ ratio:

$$
W T=\frac{\bar{W}}{\overline{\tau_{s}}}, \bar{W}=\frac{W}{\operatorname{Max}(W)}, \overline{\tau_{s}}=\frac{\tau_{s}}{\operatorname{Max}\left(\tau_{s}\right)}
$$

where $\bar{W}$ and $\overline{\tau_{s}}$ denote the normalized actuator weight and stall torque based on their maximums (i.e., Max) (see Table 2). A small WT means the high portability and capability of an actuator. The detailed comparison of Dynamixel actuators can be in footnotes ${ }^{\mathrm{c}, \mathrm{d}}$.

Table 2. Criterion of portable exoskeleton actuators [4]

\begin{tabular}{cccc}
\hline $\begin{array}{c}\text { Weights } W \\
{[\mathrm{~kg}]}\end{array}$ & $\begin{array}{c}\text { Prices } M \\
{[U S \$]}\end{array}$ & $\begin{array}{c}\text { Stall torques } \\
{[N \cdot \mathrm{m}]}\end{array}$ & Control mode \\
\hline$<0.2$ & $<500$ & $<8.0$ & Torque \\
\hline
\end{tabular}

Based on the criterion (see TABLE 2), the six series of Dynamixel actuators are compared based on the weight to torque (WT) ratios (see Eq. (1) and Fig. 2). We can see that the Dynamixel XM430 has the smallest WT (see the red diamond mark in Fig. 2) among the applicable Dynamixel actuator series (see TABLE 2). Therefore, it is chosen for the RISE exoskeleton. In spite of the above-mentioned advantages, an intrinsic drawback of Dynamixel actuators is actuation and sensing dysfunctions when subject to enduring high resistance torques. For instance, such dysfunctions may result from large impedance constant (i.e., $K_{p}$ and $K_{d}$, see Eq.(3)) control, while small impedance constants result in resistance training inefficiency (see Figs. 4 and 5). To tackle the dilemma, we propose a resistance-as-needed

${ }^{c}$ https://www.generationrobots.com/blog/en/how-to-choose-the-right-dynamixel-servomotor/
${ }^{d}$ http://www.robotis.us/dynamixel-x/ 
(RAN) controller for online impedance adaptation of the RISE exoskeleton in resistance training. Moreover, the RAN frees physiotherapists from manually adjusting exoskeleton impedance parameters for different patients, therefore greatly reducing physiotherapists' work loads and increasing rehabilitation efficiency [9].



Fig. 2. Weight to torque (WT) ratios of the applicable Dynamixel actuators (see Eq. (1) and TABLE 2).

\subsection{Resistance-as-needed ( $R A N)$ Controller}

An inverse dynamics model of the RISE exoskeleton and its wearer (see Fig. $1(\mathrm{C})$ ) is given by $[10]$ :

$$
I \ddot{q}+H(\dot{q})+G(q)=\tau+\tau_{h}+\tau_{o},
$$

where $I, H$, and $G$ represent the inertial, viscous, and gravity terms determined by the exoskeleton acceleration $\ddot{q}$, velocity $\dot{q}$, and position $q$. Note that all dynamic parameters of Eq. (2), as well as the human $\tau_{h}$ and noise $\tau_{o}$ torques are unknown.

The RAN controller is to generate the joint resistance torque $\tau$ based on a proportionalderivative $(\mathrm{PD})$ rule [11],

$$
\tau=-K_{p}(t) e(t)-K_{d}(t) \dot{e}(t)
$$

where $K_{p}(t)$ and $K_{d}(t)$ denote the impedance gains. $e(t)$ and $\dot{e}(t)$ represent the joint position and velocity errors given by,

$$
e(t)=q(t)-q_{d}(t), \dot{e}(t)=\dot{q}(t)-\dot{q}_{d}(t), \varepsilon(t)=e(t)+\beta \dot{e}(t), \beta=0.05,
$$

where $\varepsilon(t)$ denotes the joint tracking error. The RAN controller is to co-minimize the impedance efforts and motion errors of the exercise exoskeleton (see Fig. $1(\mathrm{C})$ ) over the time period $T[11]$,

$$
J_{o}(t)=J_{c}(t)+J_{p}(t), J_{c}(t)=\frac{1}{2} \int_{t-T}^{t}\left(K_{p}(t)\right)^{2}+\left(K_{d}(t)\right)^{2}, J_{p}(t)=\frac{1}{2} \int_{t-T}^{t} I(\varepsilon(t))^{2},
$$


where $I$ denotes the inertial item of the RISE exoskeleton (see Eq. (2)). The RAN cominimization leads to the exoskeleton joint impedance adaptation given by,

$$
K_{p}(t)=f(t) e(t), K_{d}(t)=f(t) \dot{e}(t), f(t)=\frac{\varepsilon(t)}{\gamma(t)}, \gamma(t)=\frac{a}{1+b \varepsilon(t)^{2}},
$$

where $\gamma(t)$ is an adaptation scalar with the positive scalars $a=0.2$ and $b=5$. All scalars, as well as the derivations of Eqs. (5) and (6) refer to our developed human-like impedance controller [11]. Online resistance adaptation is achieved by setting the desired joint position and velocity $\left(q_{d}, \dot{q}_{d}\right)$ to the initial states, i.e., $q_{d}=q_{0}, \dot{q}_{d}=0.0$ (see Fig. 3 ). The generated joint resistance torque $\tau$ increases with the increment of joint position $e$ and velocity $\dot{e}$ errors (see Eq.(6)).

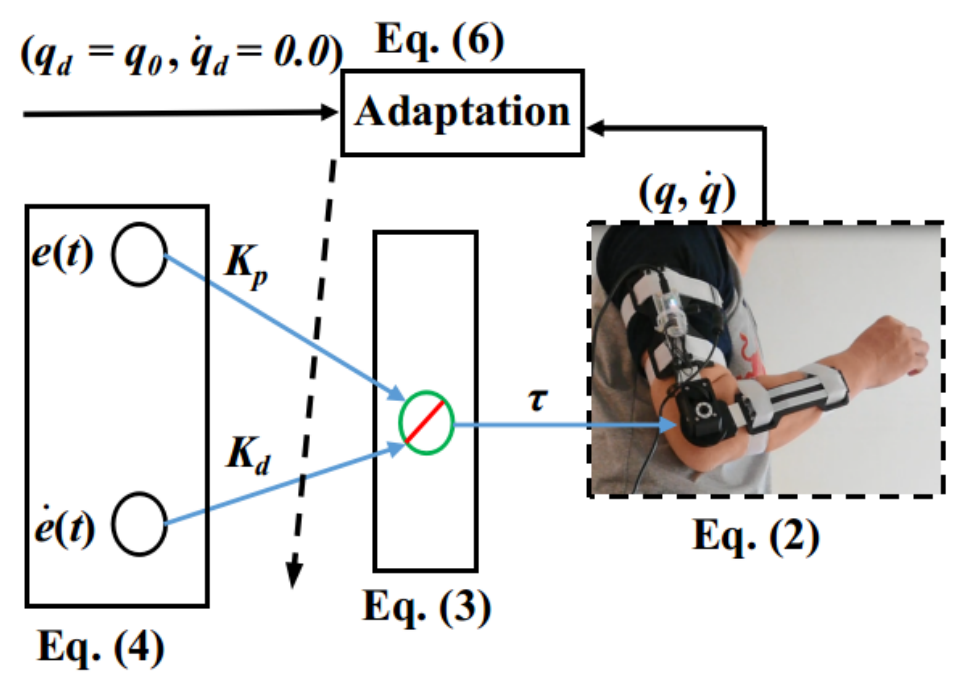

Fig. 3. Resistance-as-needed (RAN) controller (see Eqs. (3) and (6)).

\section{Experimental Results}

The RAN controller was run to control the RISE exoskeleton worn on the left arm of a healthy subject (male, 37 years old, $183 \mathrm{~cm}, 84 \mathrm{~kg}$, see Fig. 3). In the RAN, the impedance gains $K_{p}$ and $K_{d}$ are online tuned based on the joint position $e$ and $\dot{e}$ velocity errors (see Eq. (6)). The RAN controller was compared to a proportional-derivative (PD) controller with small and large impedance constants, $\left[K_{p}=0.2, K_{d}=-0.4\right]$ and $\left[K_{p}=2.0, K_{d}=\right.$ $0.5]$ in Eq. (3), respectively. The constants were chosen based on maximum and minimum impedance gains $K_{p}$ and $K_{d}$ obtained from the proposed RAN controller. The subject was asked to swing the left forearm up as much as he can, subsequently swing down to the initial position (see Fig. 4). In the periodic swings, the generated torque $\tau$ to the RISE exoskeleton should provide resistant joint motions in training (i.e., $\tau \geq 0$ ).

We can see that the proposed RAN control enables the RISE exoskeleton to generate the greater torque $\tau$ amplitude than those produced by the PD controller with the small and large impedance constants (see Fig. 4 (A)). The small impedance constants make the exoskeleton produce a negative torque (i.e., $\tau<0$, see the green line within the dotted black rectangle in Fig. $4(\mathrm{~A})$ ), when the subject swung the left forearm up. The negative torque leads to exoskeleton assistance, rather than resistance generated by the proposed RAN and large impedance control. This is because the impedance gain $K_{d}$ is negative (i.e., $K_{d}=-0.4$ ), 

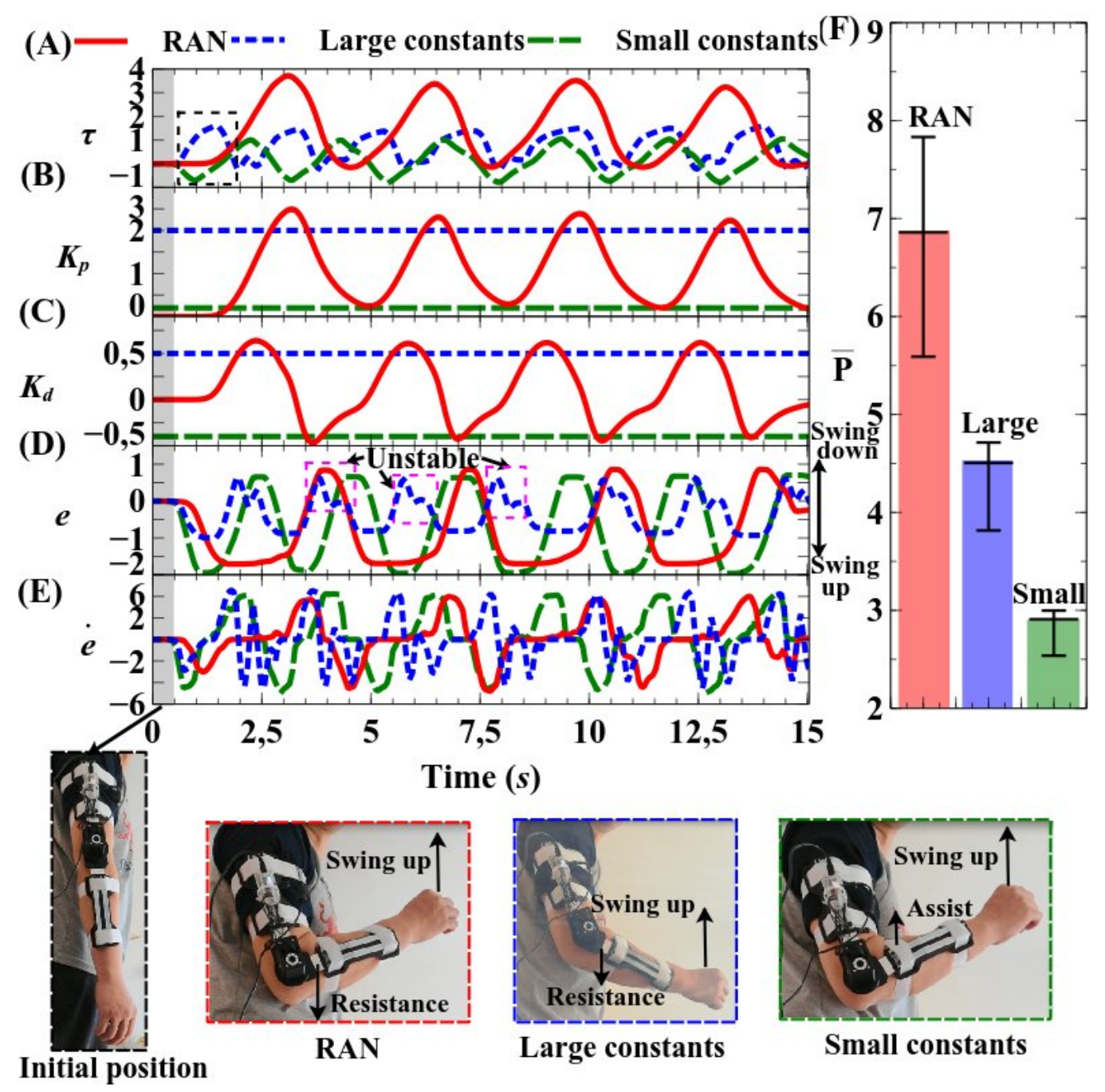

Large constants



Small constants

Fig. 4. Comparison between the proposed RAN and fixed impedance controllers (see Eqs. (6) and (3)). (A) Resistance torques $\tau$. (B), (C) Impedance gains $K_{p}$ and $K_{d}$. (D), (E) Position $e$ and velocity $\dot{e}$ errors (see Eq. (4)). (F) Statistics of the RISE exoskeleton power $\bar{P}$ (see Eq. (7)). 10 trials were run for each impedance setup. In fixed impedance control, the large and small impedance constants were set to $\left[K_{p}=2.0, K_{d}=0.5\right]$ and $\left[K_{p}=0.2, K_{d}=-0.4\right]$ in Eq. (3), respectively. Note that the resistance torque $\tau$ is highly positive, meaning great resistance. By contrast, if $\tau$ is highly negative, implying great assistance.

acting like an assistant damper with respect to swing-up motions. However, a negative impedance gain $K_{d}$ results in stable swing-down motions in the proposed RAN (see Fig. 4 (C)), compared to unstable control generated by the large impedance constant controller (e.g., see the blue line within the dotted magenta rectangles in Fig. 4 (D)). This is because the proposed RAN control provides online impedance adaptation, therefore leading to multidirectional (i.e., positive and negative) spring- and damper-like resistances in training. Such multi-directional resistances lead to variable compliant and stable exoskeleton control, that cannot be achieved by the large impedance constant control (i.e., $\left[K_{p}=2.0, K_{d}=0.5\right]$ ). The resistance training performance is quantified by the average power consumption $\bar{P}$ of the RISE exoskeleton,

$$
\bar{P}=\frac{\sum_{t=1}^{t=n} V I(t)}{n},
$$


where $V$ is the motor input voltage, i.e., $V=12.0 . I(t)$ is the motor current sensory feedback, and $n$ denotes time steps in a trial. 10 trials were run for each impedance setup. A great $\bar{P}$ means high resistance training efficiency. We can see that the proposed RAN control enables the RISE exoskeleton to consume the greatest energy (see Fig. 4 (F)), leading to the highest resistance training efficiency among the three impedance setups. This is because the RAN control provides online impedance adaptation that results in a larger torque amplitude, compared to the large and small impedance constant control.

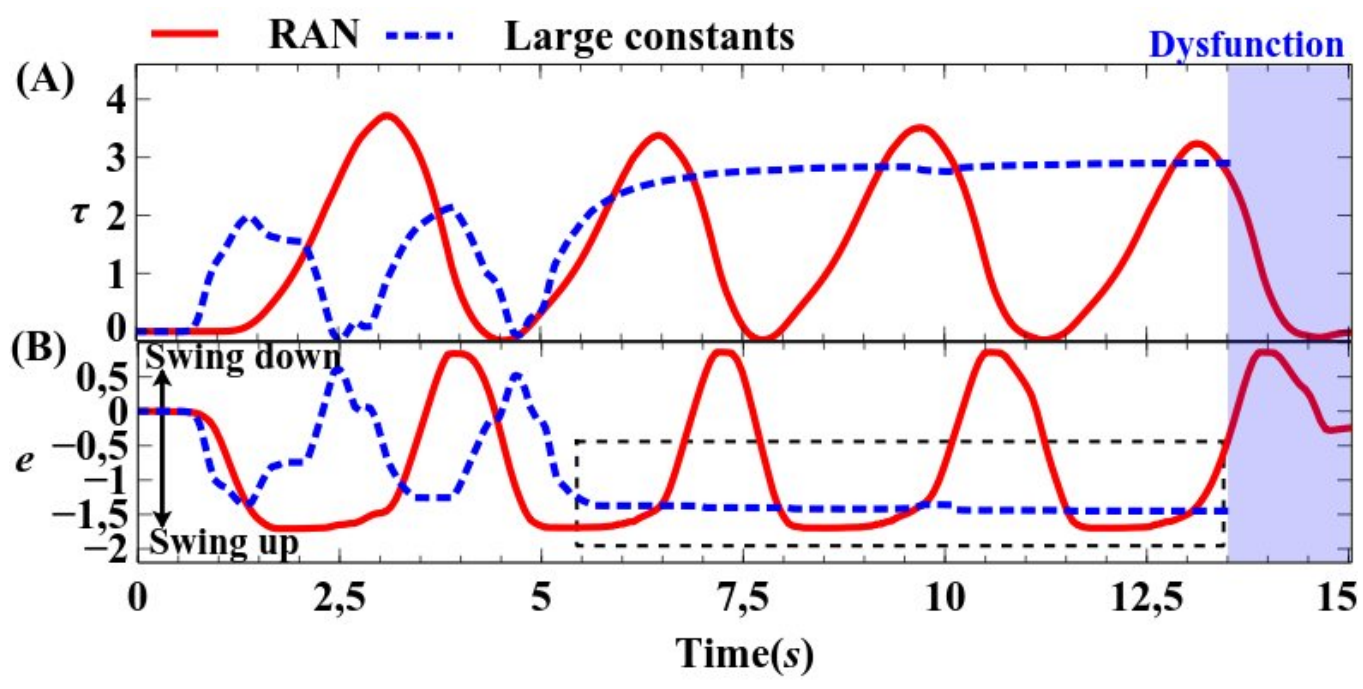

Fig. 5. Actuation and sensing dysfunction resulted from the large impedance constant control (i.e., $\left[K_{p}=\right.$ 2.0, $\left.K_{d}=0.5\right]$ ). (A) Resistance torque $\tau$ (see Eq. (3). (B) Position error $e$ (see Eq. 4). Note that the resistance torque $\tau$ is highly positive, meaning great resistance.

Surprisingly, the large impedance constant control produces the smaller resistance torque and position amplitudes than those generated by the proposed RAN (see Fig. 4 (A) and (D)). This is because the subject was asked to try a large forearm swing-up amplitude (see the blue line within the dotted black rectangle in Fig. 5 (B)), but it leads to the actuation and sensing dysfunction. This is an intrinsic drawback of the chosen Dynamixel actuator when subject to enduring the high resistance torque $\tau$ (see the dotted blue line in Fig. 5 (A)). The footnote ${ }^{\mathrm{e}}$ provides a link to the video.

\section{Discussion and Conclusion}

Generally, the exercise exoskeleton actuation can be categorized as passive, active, and hybrid. Physical compliant mechanisms (e.g., springs) are usually used for compliant resisters in training. For instance, a senseless arm exoskeleton ${ }^{f}$ is passively actuated for resistance training. Such fixed actuation has an intrinsic dilemma where small and high impedance actuation may decrease resistance training efficiency and wearers' comfortability. Simple passive actuation decreases resistance adaptivity, while complicated active actuation increases exoskeleton complexity. Active actuation implementation relies heavily on external sensing such as force and EMG sensing [10]. Such sensing is intrinsically noisy and subject to environmental changes. For instance, the external sensing becomes inaccurate when wearers sweat and sensors fall out. In hybrid actuation, passive and active mechanisms are applied

${ }_{\mathrm{e}}^{\mathrm{e}} \mathrm{https} / / /$ www.youtube.com/watch?v=qU8EU-6qbM4

${ }_{\mathrm{f}}^{\mathrm{h}}$ ttps://xotrainer.com/products/armore-wearable-arm-exerciser 
to different exoskeleton joints. However, such hybrid actuation inherits inherent drawbacks of passive and active mechanisms. Taken together, existing actuation designs are too simple or complex to control adaptive and portable exoskeletons in at-home resistance training. For instance, Wu et al. (2014) utilized pneumatic muscles (PMs) to actuate a ground-based exoskeleton in resistance training [6]. A major disadvantage of the PMs is their low usage efficiency. They require additional air suppliers that raise usage costs. To increase exoskeleton's efficiency and portability, Manna and Dubey (2017) applied a lightweight (i.e., 0.21 $\mathrm{kg}$ ) DC motor to a $1.8 \mathrm{~kg}$ elbow exoskeleton [7]. However, its resistant impedance and torque are fixed and very small (i.e., $\leq 0.035 N \cdot m$ ), leading to low resistance training adaptivity and efficiency. To increase the adaptivity, Liu et al. (2019) designed a variable stiffness actuator (VSA) to drive a $3.1 \mathrm{~kg}$ arm exoskeleton [8]. However, its implementation relies heavily on physical compliant mechanisms (e.g., springs) and external (i.e., force) sensing, therefore leading to high mechatronic complexities and sensing dependence. To address the problem, we propose a software-based resistance adaptation mechanism where the impedance gains $K_{p}$ and $K_{d}$ are online tuned (see Eqs.(3) and (6)). Moreover, the online resistance adaptation replies only on internal position and velocity feedback, rather than external (e.g., EMG) feedback and physical compliant mechanisms (e.g., springs) [8,10]. Note that the focused topic in this paper is resistance control of exercise exoskeletons, rather than assisted-as-needed controllers of assistive exoskeletons [10]. Extensive reviews on exoskeleton design and control can be seen in $[4,5]$. Taken together, the proposed RAN controller and RISE exoskeleton pave a way for portable and affordable at-home resistance training.

In spite of the presented effectiveness, there will be interesting extensions of the proposed RAN control and RISE exoskeleton in future. First, the current arm design will be extended to a shoulder-arm one. Second, a physiological means will be utilized to quantified the exercise effects of specific muscles.

\section{Acknowledgments}

This research is supported by the Human Frontier Science Program (Grant no. RGP0002/2017, P.M. = Project Co-PI) and a startup grant on bio-inspired robotics from the Vidyasirimedhi Institute of Science and Technology (VISTEC, P.M. = Project PI). The authors would like to thank Cao Danh Do, John Hallam, and Anders Lyhne Christensen for their help and consultation.

\section{References}

1. L. Di Pollina, I. Guessous, V. Petoud, C. Combescure, B. Buchs, P. Schaller, M. Kossovsky and J.-M. Gaspoz, BMC Geriatrics 17, p. 53 (2017).

2. R. Lewis, C. B. Gómez Álvarez, M. Rayman, S. Lanham-New, A. Woolf and A. Mobasheri, BMC Musculoskeletal Disorders 20, p. 164 (2019).

3. E. G. Ciolac and J. M. Rodrigues-da Silva, Sports Medicine 46, 1239 (2016).

4. S. K. Manna and V. N. Dubey, Medical Engineering \& Physics 60, 1 (2018).

5. T. Proietti, V. Crocher, A. Roby-Brami and N. Jarrassé, IEEE Reviews in Biomedical Engineering 9, 4 (2016).

6. T.-M. Wu and D.-Z. Chen, Journal of rehabilitation research and development 51, 111 (2014).

7. S. K. Manna and V. N. Dubey, 2017 International Conference on Rehabilitation Robotics (ICORR), 2017.

8. Y. Liu, S. Guo and Z. Yang, 2019 IEEE International Conference on Mechatronics and Automation (ICMA), aug 2019.

9. A. Stephenson and J. Stephens, Disability and Rehabilitation: Assistive Technology 13, 245(apr 2018).

10. T. Teramae, T. Noda and J. Morimoto, IEEE Robotics and Automation Letters 3, 210 (2018).

11. X. Xiong and P. Manoonpong, IEEE International Conference on Robotics and Biomimetics, (Kuala Lumpur, 2018). 